\title{
How to reduce complications and sequalae in rhinoplasty
}

\author{
Moo Hyun Paik \\ Department of Plastic and Reconstructive Surgery, Agape Christian Hospital, Ulaanbaatar, Mongolia
}

In recent years, the popularity of cosmetic surgery and the demand for such procedures have dramatically increased, and questions about aesthetic rhinoplasty are particularly common. Because the results of rhinoplasty procedures are readily visible, rhinoplasty is particularly susceptible to critical analyses. With the increasing number of surgical procedures, the incidence of complications has also increased, as surgery and complications thereof are two sides of the same coin. Thus, we are faced with the urgent question of how to reduce the complication rate. Instead of the technical aspects of surgery, I would like to discuss our attitudes and behaviors as plastic surgeons. In particular, we should adhere to the following guidelines.

Meet with patients directly. We can only deliver what patients want by accurately assessing their needs through direct consultations and by considering those needs when formulating surgical plans.

Monitor the treatment and its progress. This enables the early detection of any emerging problems and prevents the onset of complications. When a problem is detected, appropriate therapy should be started without hesitation.

If hematoma is suspected, open the affected site immediately. Hematoma removal can minimize inflammation, promote successful graft integration, and prevent excessive scar formation. Without appropriate interventions, pressure from a hematoma can reduce circulation to the area, leading to skin necrosis.

If inflammation is suspected, open the affected site immedi-

Correspondence: Moo Hyun Paik

Department of Plastic and Reconstructive Surgery, Agape Christian Hospital,

\#101 Building 38, Nisekh, 10th Khoroo, Khan-Uul district, Ulaanbaatar, Mongolia

E-mail:mhpaik305@naver.com

Received June 3, 2019 / Revised June 5, 2019 / Accepted June 7, 2019 ately. It is better to remove any questionable prostheses and grafts as soon as possible. A "wait-and-see" approach is never appropriate, because the actual situation inside the body can be much more severe than the patient's external appearance would suggest.

Do not insert any prostheses, except in the area of the nasal bridge. Prostheses in the nasal tip, septum, and columella nasi are prone to creating ongoing problems in the future.

Avoid using new materials. Time is needed for the safety of a new product to be proven. Examples include Medpor, resorbable plates or mesh, Alloderm blocks, and non-resorbable fillers. Do not blindly follow others in using such materials or techniques; instead, wait until their safety has been proven.

Exposed prostheses should be promptly removed. First, allow the wound to heal after removing the prosthesis, and then perform the main surgical procedure. Although it is possible to remove a prosthesis after healing, doing so requires extensive experience, so removing the prosthesis early should be the standard of care.

Perform irreversible procedures with minimal invasiveness. Examples include hump reduction, ala reduction, and cephalic trimming of the lateral crus. Resecting structures unnecessarily in an attempt to achieve immediate results can make restorations difficult if any complications arise and can cause considerable suffering to the patient.

Plan the operation according to the physical status of the patient's nose. It is better to perform safe operations with lasting results and to appropriately manage patients' expectations prior to surgery. Even if patients demand unachievable outcomes, surgeons should be able to explain the outcomes that may be re- 
alistically expected and perform surgery within safe boundaries. Select procedures that you can handle. It is better not to start operations that are unreasonably challenging. Do not blindly accept the cases presented at academic conferences, because those cases are generally only the most successful ones, with the best results. Forcing oneself to complete unreasonable operations can cause surgical failure, loss of confidence, and an adverse effect on self-improvement. It hurts both the surgeon and the patient.

Improve yourself. Select surgical procedures that you can handle, thoroughly prepare yourself, and try your best to succeed. Successful results can foster your self-confidence, and you will soon find yourself being able to handle new cases adequately. Success from your best efforts will feed positively into your next challenge.

\section{NOTES}

\section{Conflict of interest}

No potential conflict of interest relevant to this article was reported.

\section{ORCID}

Moo Hyun Paik https://orcid.org/0000-0003-3586-1727 